\title{
Erratum to: Sequence differences in the seed dormancy gene Qsd1 among various wheat genomes
}

Kazumitsu Onishi ${ }^{1}$, Miki Yamane ${ }^{2}$, Nami Yamaji ${ }^{2}$, Mayumi Tokui ${ }^{1}$, Hiroyuki Kanamori ${ }^{3}$, Jianzhong $\mathrm{Wu}^{3}$, Takao Komatsuda ${ }^{3}$ and Kazuhiro Sato ${ }^{2^{*}}$

\section{Erratum}

After the publication of this article [1] the authors noted that Fig. 2 was incorrect and a number of species abbreviations were not in their intended positions, such as At2 and Bd2.

A correct version of Fig. 2 is included with this Erratum.

The original article has been corrected.

\author{
Author details \\ 'Obihiro University of Agriculture and Veterinary Medicine, Obihiro 080-8555, \\ Japan. ${ }^{2}$ Institute of Plant Science and Resources, Okayama University, \\ Kurashiki 710-0046, Japan. ${ }^{3}$ Institute of Crop Science, National Agriculture \\ and Food Research Organization, Tsukuba 305-8634, Japan.
}

Received: 10 August 2017 Accepted: 10 August 2017

Published online: 29 August 2017

\section{Reference}

1. Onishi K, Yamane M, Yamaji N, Tokui M, Kanamori H, Wu J, Komatsuda T,

Sato K. Sequence differences in the seed dormancy gene Qsd1 among various wheat genomes. BMC Genomics. 2017;18:497. doi:10.1186/s12864017-3880-6.

\footnotetext{
* Correspondence: kazsato@rib.okayama-u.ac.jp

${ }^{2}$ Institute of Plant Science and Resources, Okayama University, Kurashiki

710-0046, Japan

Full list of author information is available at the end of the article
} 


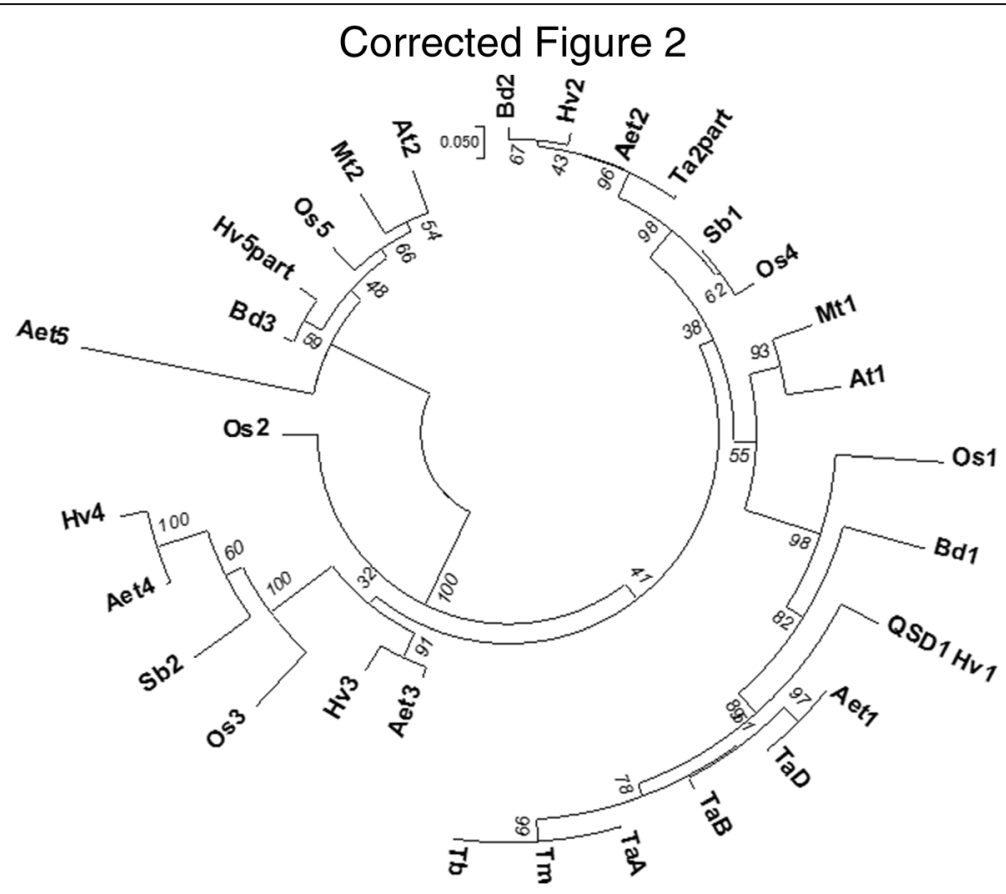

Fig. 2 Multiple alignment of QSD1 amino acid sequences (Hv1: Haruna Nijo). The listed sequences are from accessions showing similarity to QSD1 (E-value < E-145) by Blastp analysis of NCBI nr. Species and homologs (with accession numbers) are as follows: Hordeum vulgare: Hv1 (BAK04026.1), Hv2 (BAK07780.1), Hv3 (P52894.1), Hv4 (BAK05632.1), Hv5part (BAJ90574.1); Triticum aestivum: TaA (AK333743.1), TaB (LC209618), TaD (LC209619), Ta2part (CAE54279.1); T. monococcum: Tm (LC209623); T. boeoticum: Tb (LC209622); Aegilops tauschii: Aet1 (EMT25616.1), Aet2 (EMT05433.1), Aet3 (EMT23015.1), Aet4 (EMT08497.1), Aet5EMT29455.1); Oryza sativa: Os1 (NP_001063248.1), Os2 (NP_001064504.1) Os3 (NP_001064505.2), Os4 (NP_001060284.1), Os5 (NP_001058716.1); Sorghum bicolor: Sb1 (XP_002463187.1), Sb2 (XP_002467302.1); Brachypodium distachyon: Bd1 (XP_003578159.1), Bd2 (XP_010235387.1), Bd3 (XP_003557680.1); Arabidopsis thaliana: At1 (NP_173173.3), At2 (AAK68842.1); Medicago truncatula: Mt1 (XP_003627448.1), Mt2 (XP_003613139.1). Scale bar indicates Poisson Correction distance. Numerals show test values for 1000 bootstrap replications 